\title{
ONE DIMENSIONAL PERTURBATIONS OF COMPACT OPERATORS
}

\author{
HARRY HOCHSTADT ${ }^{1}$
}

\begin{abstract}
Let $K$ denote a compact selfadjoint operator acting on a Hilbert space $H$. $L$ denotes a one dimensional selfadjoint operator also acting on $H$. It is shown that the eigenvalues of $K$ and $K+L$ interlace on the real axis.
\end{abstract}

The purpose of this article is to prove the following theorem.

THEOREM. Let $K$ denote a compact, selfadjoint operator acting on a Hilbert space $H$. We assume that the nullspace of $K$ is empty. Let $L$ denote a one dimensional, selfadjoint operator, also acting on $H$. Between every pair of distinct, successive eigenvalues $\left(\lambda_{i}, \lambda_{i+1}\right)$ of $K$ there is precisely one eigenvalue of $K+L$ in one of the intervals $\left[\lambda_{i}, \lambda_{i+1}\right)$ or $\left(\lambda_{i}, \lambda_{i+1}\right]$ or $\left(\lambda_{i}, \lambda_{i+1}\right)$. Every eigenvalue of multiplicity $n>1$ of $K$ is also an eigenvalue of $K+L$, of multiplicity $n$ or $n-1$.

The above theorem may be viewed as a generalization of certain theorems regarding second order differential equations. Consider, for example,

$$
y^{\prime \prime}+(\lambda-q(x)) y=0
$$

where $q(x)$ is real and continuous on $[0,1]$, subject to the boundary conditions

(a) $y(0)=0, y(1)=0$,

(b) $y(0)=0, y^{\prime}(1)=0$.

The eigenvalues of (1)(a) and (1)(b) are real and alternate on the real axis. This fact is known. It is however an immediate consequence of the above theorem. Both problems (1)(a) and (1)(b) can be investigated by converting the differential operators into compact integral operators. The latter differ by a one dimensional operator so that the above theorem can be invoked.

\footnotetext{
Received by the editors June 19, 1972.
}

AMS (MOS) subject classifications (1970). Primary 47A55, 47B05; Secondary 34B25.

${ }^{1}$ This research was supported by the National Science Foundation under grant GP 27960. 
ProOF of TheOrem. We can associate with $K[1]$ a complete orthonormal set $\left\{\phi_{i}\right\}$ such that each $\phi_{i}$ is an eigenfunction of $K$ so that

$$
K \phi_{i}=\lambda_{i} \phi_{i}
$$

and $\lambda_{i} \neq 0$ for all $i$, by hypothesis.

Since $L$ is one dimensional we can select an arbitrary element $\Psi$ in the range of $L$, as the basis for that subspace. In fact $L$ can now be represented in the form

$$
L \phi=\alpha \Psi(\phi, \Psi)
$$

where $\alpha$ is real. If $\Psi$ is so chosen that $\|\Psi\|=1, \alpha$ will be the only nonzero eigenvalue of $L$.

The eigenvalues and eigenfunctions of $K+L$ will have to satisfy the equation

$$
K \phi+L \phi=\lambda \phi \text {. }
$$

To investigate the above equation we shall expand both $\phi$ and $\Psi$ in terms of the complete orthonormal set $\left\{\phi_{i}\right\}$,

$$
\phi=\sum_{i=1}^{\infty} f_{i} \phi_{i}, \quad \Psi=\sum_{i=1}^{\infty} g_{i} \phi_{i} .
$$

Next we insert (5) in (4) and obtain

or equivalently

$$
\sum_{i=1}^{\infty} f_{i} \lambda_{i} \phi_{i}+\alpha \sum_{i=1}^{\infty} g_{i} \phi_{i}\left(\sum_{i=1}^{\infty} f_{i} \bar{g}_{i}\right)=\lambda \sum_{i=1}^{\infty} f_{i} \phi_{i}
$$

$$
\lambda_{i} f_{i}+\alpha g_{i} \sum_{i=1}^{\infty} f_{i} \bar{g}_{i}=\lambda f_{i}, \quad i=1,2, \cdots
$$

We shall assume, for the moment, that all eigenvalues of $K$ are simple and that $g_{i} \neq 0$ for all $i$. (7) can now be rewritten in the form

$$
f_{i}=\propto \frac{g_{i}}{\lambda-\lambda_{i}} \sum_{i=1}^{\infty} f_{i} \bar{g}_{i} .
$$

$\sum_{i=1}^{\infty} f_{i} \bar{g}_{i}$ cannot vanish. Otherwise some eigenfunction $\phi$ of $K+L$ would be orthogonal to $\Psi$. In that case $L \phi=0$ and (4) would show that $\phi$ is an eigenfunction of $K$ and therefore one of the $\phi_{i}$. Then the corresponding coefficient $g_{i}$ in (5) would vanish. But we assume that no $g_{i}$ vanishes. We now multiply (8) by $\bar{g}_{i}$, sum over all $i$ and thus obtain

$$
\sum_{i=1}^{\infty} \frac{\left|g_{i}\right|^{2}}{\lambda-\lambda_{i}}=\frac{1}{\alpha} .
$$


Every eigenvalue $\lambda$ of $K+L$ must satisfy (9). Viewing the left side as a function of $\lambda$ we see that it has poles at all $\lambda_{i}$ and is of negative slope for all other real $\lambda$. It follows that between two successive $\lambda_{i}(9)$ has precisely one solution. In turn if $\lambda$ satisfies (9) we can solve (7) for $f_{i}$ and thus obtain an eigenfunction of $K+L$ corresponding to $\lambda$. Thus the theorem is proved under the restrictions stated earlier.

We can now consider the case where one of the $g_{i}$ vanishes. In that case (7) shows that $\lambda=\lambda_{i}$ is an eigenvalue of $K+L$. The other eigenvalues must still satisfy (9), but only the nonvanishing terms in the sum in (9) play a role. Again we see that the theorem is true.

Finally we consider the possibility that some eigenvalues of $K$ have multiplicity $n>1$. If all eigenfunctions $\phi_{i}$ corresponding to such an eigenvalue are orthogonal to $\Psi$, the corresponding $g_{i}$ in (7) vanish and $\lambda=\lambda_{i}$ will be an eigenvalue of $K+L$ of multiplicity $n$. Suppose $\tilde{\lambda}$ is an eigenvalue of $K$ of multiplicity $n$ and w.l.o.g. $\phi_{1}, \phi_{2}, \cdots, \phi_{n}$ the corresponding eigenfunctions. Again w.l.o.g. suppose that $\left(\phi_{1}, \Psi\right) \neq 0$. Each of these $\phi_{i}$ can be represented in the form

$$
\phi=\rho_{i} \Psi+\eta_{i}
$$

such that $\rho_{1} \neq 0$ and $\left(\Psi, \eta_{i}\right)=0, i=1,2, \cdots, n$.

We now let

$$
\phi=\sum_{i=1}^{n} \beta_{i} \phi_{i}
$$

and insert this $\phi$ in (4) with $\lambda=\tilde{\lambda}$. Since $K \phi=\tilde{\lambda} \phi$ the latter reduces to

$$
L \phi=\alpha \Psi \sum_{i=1}^{n} \beta_{i} \rho_{i}\|\Psi\|^{2}=0
$$

and the above equation has at least $n-1$ linearly independent solutions, which when inserted in (11) yield $n-1$ linearly independent eigenfunctions of $K+L$ corresponding to the eigenvalue $\tilde{\lambda}$. This completes the proof of the theorem.

\section{REFERENCES}

1. F. Riesz and B. Sz.-Nagy, Leçons d'analyse fonctionnelle, Akad. Kiadó, Budapest, 1953; English transl., Ungar, New York, 1955, p. 234. MR 15, 132; MR 17, 175.

Department of Mathematics, Polytechnic Institute of Brooklyn, Brooklyn, NEW YORK 11201 